\title{
Robust Estimation and Tracking of Power System Harmonics Using an Optimal Finite Impulse Response Filter
}

\author{
Bo-kyu Kwon ${ }^{1}$, Soohee Han ${ }^{2, *}$ and Kwang Y. Lee ${ }^{3,+}$ \\ 1 Department of Control and Instrumentation Engineering, Kangwon National University, \\ Gangwon-do 24341, Korea; bkkwon@kangwon.ac.kr \\ 2 Department of Creative IT Engineering, Pohang University of Science and Technology (POSTECH), Pohang, \\ Gyeongbuk 37673, Korea \\ 3 Department of Electrical and Computer Engineering, Baylor University, Waco, TX 76798, USA; \\ Kwang_Y_lee@baylor.edu \\ * Correspondence: sooheehan@postech.ac.kr; Tel.: +82-10-4660-7140 \\ + Current address: Department of Creative IT Engineering, Pohang University of Science and Technology \\ (POSTECH), Pohang, Gyeongbuk 37673, Korea.
}

Received: 30 May 2018; Accepted: 9 July 2018; Published: 11 July 2018

\begin{abstract}
In this paper, a robust estimation method for estimating the power system harmonics is proposed by using the optimal finite impulse response (FIR) filter. The optimal FIR filter is applied to the state space representation of the noisy current or voltage signal and estimates the magnitude and phase-angle of the harmonic components. Due to the FIR structure, the FIR filter is more robust against model uncertainty than the Kalman filter. Hence, the FIR filter-based method will give a more robust solution for the power system harmonic estimation than the previous Kalman filter-based approaches. The performance and robustness of the proposed method are verified through simulation. Moreover, the proposed method is employed in the power conditioning system to estimate the harmonic components and total harmonic distortions.
\end{abstract}

Keywords: robust harmonics estimation; power system harmonics; optimal FIR filter; power conditioning system; total harmonic distortions

\section{Introduction}

Non-linear loads connected to the power system have led to increased harmonic levels, and these harmonics result in serious problems related to the power system operation such as voltage distortion, increased losses and heating [1-4]. Hence, due to the significantly increasing use of power electronic devices, power quality has become an important issue in the power system operation. Power system harmonics are one of the important indices of the quality of power, and thus, it is necessary to estimate the power system harmonic components to provide high-quality of power [5-8].

In many power system applications, fast Fourier transform (FFT) and discrete Fourier transform (DFT) have been extensively used to estimate the harmonic components of voltage or current signals owing to its fast computation and simplicity [9-12]. Both techniques give an exact estimation of the harmonic components for the stationary noiseless signals. However, FFT and DFT do not consider the noise in their derivations and are suitable for a stationary signal, these techniques may lose accuracy and have poor performance for the non-stationary and noisy signals.

In order to suppress the noise and deal with the time varying harmonics, the Kalman filter-based approaches have been suggested with state space representation of the noisy signal [13-15]. In these approaches, the harmonic components are represented as state variables and are estimated from the Kalman filter. Since the Kalman filter gives the optimal estimation result, these techniques are 
considered as effective methods for estimating the time varying harmonics of the power system. However, these methods essentially require the prior information of the system and signals such as initial harmonic components and stochastic information of the variance of harmonics and the measurement noise. However, it is hard to acquire their information reliably. Especially, the optimality of the Kalman filter depends on the accuracy of the process and measurement noise covariance matrices. Small values of the process and measurement noise covariance matrices yield unreliable estimates, and large values could produce the divergence of the estimation result. Thus, Kalman filter-based approaches may have poor performance or even diverge with these uncertainties. In the state space representation of the noisy signal, the variance of harmonic components is assumed as a random walk motion and is considered as the process noise with an unknown noise covariance matrix. In real cases, it is very hard to acquire the exact information of the variance of harmonic components. To this end, the selection of the process and measurement noise covariance matrices is a critical issue for the use of the Kalman filter. In order to solve these problems, modified Kalman filter-based harmonics estimation techniques have been proposed [2,4,8,16-23]. In [16], it was demonstrated that the estimation result depends more on the ratio of the process and measurement noise covariance matrices than on each of their values. The use of two process noise covariance matrices for dynamic harmonic tracking estimation was proposed in [17]. In [19], a method for self-tuning of the model error covariance was presented to track harmonic fluctuations. The adaptive Kalman filter-based methods were proposed in $[17,18,22,23]$. In [18], the adaptive Kalman filter that switches between two basic process noise covariance matrices' model for steady-state and transient estimation was applied for the dynamic harmonic state estimation and harmonic injection tracking. A method for adaptive tuning of the process and measurement noise covariance matrices was proposed in [23].

However, since the Kalman filter is the infinite impulse response (IIR) structured filter, which makes use of all information from the initial time to the current time and has an internal state, undesirable uncertainties can accumulate in the states. If the error dynamics of the Kalman filter is marginally stable, small errors can be the cause of the divergence problem. Thus, the estimated harmonics of Kalman filter-based approaches may diverge for the systems with modeling errors or numerical errors. Moreover, it takes a long time to get back to a real state even if temporary undesirable errors disappear. Therefore, the robust estimation method for power system harmonics is required to overcome the demerits of the Kalman filter-based harmonic estimation method. In the signal processing area, the receding horizon or moving horizon estimators with a finite impulse response (FIR) structure have been proposed as an alternative to the IIR-structured estimators such as the Kalman filter [24-28]. Since the FIR-structured filters use the recent finite information, they guarantee bounded input bounded output (BIBO) stability and have more robustness and a faster response than IIR-structured filters. Moreover, FIR estimators are less sensitive to the process noise than IIR estimators [25]. Hence, the FIR filter could be employed for the robust estimation of power system harmonics instead of using the Kalman filter.

In this paper, we propose the robust estimation method for power system harmonics using the optimal FIR filter. The noisy current or voltage signal is represented as a state space model, the harmonic components of signal are represented as state variables, and then, an optimal FIR filter is applied to estimate the magnitude and phase-angle of harmonic components. The contribution of this paper is as follows:

- Since the optimal FIR filter used in the proposed method consists of the linear combination of a finite number of past measurements without feedback information, the stability of the proposed method is guaranteed in the sense of bounded input and bounded output (BIBO) stability. Thus, the proposed method can avoid the divergence problem that may arise in the case of Kalman filter-based approaches.

- The proposed method can give more robustness than Kalman filter-based approaches with respect to the uncertainties, because the optimal FIR filter is less sensitive to the process noise than an IIR-structured filter, such as the Kalman filter. 
- The proposed method makes estimation more robust to temporary uncertainties and numerical errors compared with Kalman filter-based methods.

- Since the optimal FIR filter used in this approach does not need any a priori information of the initial state [27], the proposed harmonic estimation method can prevent errors that come from the unknown initial state.

- Since the optimal FIR filter used in the proposed method is designed to have a deadbeat property, the proposed method could provide a fast tracking ability.

- Since the optimal FIR filter in the proposed method is a time-invariant filter, the proposed method can provide better computational efficiency than the Kalman filter-based approaches, which use the time-varying Kalman filter.

This paper is organized as follows: In Section 2, state space models for noisy current or voltage signals and the robust estimation method for power system harmonics are proposed by using the optimal FIR filter. In Section 3, the performances of the proposed scheme and the Kalman filter-based approaches are compared with the signal that has the time-invariant and time-varying harmonic components. In Section 4, the proposed method will be employed in the power conditioning system to estimate the harmonic components and total harmonic distortions. Finally, the conclusion is presented in Section 5.

\section{Robust Estimation of Power System Harmonics Using an Optimal FIR Filter}

A current or voltage signal that includes harmonic components can be represented with measurement noise as:

$$
y(t)=\sum_{m=1}^{M} a_{m, t} \cos \left(\omega m t+\theta_{m, t}\right)+v_{t}
$$

where $m$ is the harmonic order and $a_{m, t}$ and $\theta_{m, t}$ are the magnitude and phase-angle of the $m$-th harmonic component at time $t$, respectively. $\omega$ is the fundamental power system frequency, and $v_{t}$ is the measurement noise, which is a zero-mean white Gaussian random process with the covariance $R^{s}$.

Let the sampling time interval be $\Delta t=t_{k+1}-t_{k}$ and the time index $k$ be the $k$-th sampling time $\left(t_{k}=\Delta t k\right)$, then the signal can be expressed at time $k$ as:

$$
y_{k}=\sum_{m=1}^{M} a_{m, k} \cos \left(\omega m \Delta t k+\theta_{m, k}\right)+v_{k}
$$

The signal (2) can be represented as a linear time-invariant state space model as follows.

If it is assumed that the variance of the magnitude of the $m$-th harmonic components $\Delta a_{m, k}(m=1, \cdots, M)$ follows a random walk motion, then the magnitude of the $m$-th harmonic components at time $k+1$ can be represented as:

$$
a_{m, k+1}=a_{m, k}+\Delta a_{m, k}
$$

Let the state variables $x_{k}$ be:

$$
x_{k}=\left[\begin{array}{c}
x_{1, k} \\
x_{2, k} \\
\vdots \\
x_{2 M-1, k} \\
x_{2 M, k}
\end{array}\right]=\left[\begin{array}{c}
a_{1, k} \cos \left(\omega \Delta t k+\theta_{1, k}\right) \\
a_{1, k} \sin \left(\omega \Delta t k+\theta_{1, k}\right) \\
\vdots \\
a_{M, k} \cos \left(\omega M \Delta t k+\theta_{M, k}\right) \\
a_{M, k} \sin \left(\omega M \Delta t k+\theta_{M, k}\right)
\end{array}\right]
$$


then, the signal (2) at time $k+1$ can be represented as:

$$
\begin{aligned}
y_{k+1}= & \sum_{m=1}^{M} x_{2 m-1, k+1}+v_{k+1} \\
= & \sum_{m=1}^{M} a_{m, k+1} \cos \left(\omega m \Delta t k+\omega m \Delta t+\theta_{m, k}\right)+v_{k+1} \\
= & \sum_{m=1}^{M}\left[( a _ { m , k } + \Delta a _ { m , k } ) \left(\cos (\omega m \Delta t) \cos \left(\omega m \Delta t k+\theta_{m, k}\right)\right.\right. \\
& \left.-\sin (\omega \Delta t k) \sin \left(\omega m \Delta t k+\theta_{m, k}\right)\right]+v_{k+1} \\
= & \sum_{m=1}^{M}\left[\cos (\omega m \Delta t) x_{2 m-1, k}-\sin (\omega m \Delta t) x_{2 m, k}\right]+w_{1, k}+v_{k+1} .
\end{aligned}
$$

where $w_{1, k}=\sum_{m=1}^{M} \Delta a_{m, k} \cos \left(\omega m \Delta t k+\omega m \Delta t+\theta_{m, k}\right)$. Additionally,

$$
\begin{aligned}
& \sum_{m=1}^{M} x_{2 m, k+1} \\
= & \sum_{m=1}^{M} a_{m, k+1} \sin \left(\omega m \Delta t k+\omega m \Delta t+\theta_{m, k}\right) \\
= & \sum_{m=1}^{M}\left[\left(a_{m, k}+\Delta a_{m, k}\right)(\sin (\omega m \Delta t) \cos (\omega m \Delta t k\right. \\
& \left.\left.+\theta_{m, k}\right)+\cos (\omega \Delta t k) \sin \left(\omega m \Delta t k+\theta_{m, k}\right)\right] \\
= & \sum_{m=1}^{M}\left[\sin (\omega m \Delta t) x_{2 m-1, k}+\cos (\omega m \Delta t) x_{2 m, k}\right]+w_{2, k}
\end{aligned}
$$

where $w_{2, k}=\sum_{m=1}^{M} \Delta a_{m, k} \sin \left(\omega m \Delta t k+\omega m \Delta t+\theta_{m, k}\right)$.

From (4)-(6), the time-invariant state space model for Signal (2) can be represented as:

$$
\begin{aligned}
x_{k+1} & =\Phi x_{k}+w_{k}^{a} \\
y_{k} & =H x_{k}+v_{k},
\end{aligned}
$$

where:

$$
\begin{aligned}
\Phi= & \text { blkdiag }\left[\begin{array}{cc}
\cos (\omega m \Delta t) & -\sin (\omega m \Delta t) \\
\sin (\omega m \Delta t) & \cos (\omega m \Delta t)
\end{array}\right], \\
& (m=1, \cdots, M), \\
H= & {\left[\begin{array}{lllll}
1 & 0 & \cdots & 1 & 0
\end{array}\right], }
\end{aligned}
$$

where blkdiag in (9) means the block diagonal matrix composed of its parameters and $w_{k}^{a}=\left[w_{1, k}^{T} w_{2, k}^{T}\right]^{T}$ assumed as the white Gaussian random process noise with time-varying covariance $Q_{k}^{t i}$.

Since the increments of the harmonic components $\Delta a_{m, k}$ in (3) is unknown and time-varying, $w_{k}^{a}$ and its covariance $Q_{k}^{t i}$ in (7) are also unknown and time-varying variables. Therefore, the state estimator for the system (7) and (8) should be robust to the unknown process noise. The FIR filter is less sensitive for process noise than the Kalman filter; hence, the FIR filter-based method for power system harmonic estimation may give a more robust solution than the Kalman filter-based method.

In order to design the optimal FIR filter, consider the following state space model:

$$
\begin{aligned}
x_{k+1} & =A x_{k}+G w_{k}, \\
y_{k} & =C x_{k}+v_{k},
\end{aligned}
$$


where $x_{k}$ is the state vector, $y_{k}$ is the measurement and $w_{k}$ and $v_{k}$ are the process noise and measurement noise, respectively. $w_{k}$ and $v_{k}$ are white Gaussian random noises with covariance $Q$ and $R$, respectively.

The optimal FIR filter can be designed as a linear function of finite measurements on the horizon $[k-N, k-1]$ :

$$
\hat{x}_{k \mid k-1}=L Y_{k-1}
$$

where $L$ is the optimal filter gain, which is given by:

$$
L=\left[\begin{array}{llll}
l_{0} & l_{1} & \cdots & l_{N-1}
\end{array}\right]
$$

and $Y_{k-1}$ is the finite number of measurements on the horizon as:

$$
Y_{k-1}=\left[\begin{array}{llll}
y_{k-N}^{T} & y_{k-N+1}^{T} & \cdots & y_{k-1}^{T}
\end{array}\right] .
$$

For the state space model (11) and (12), the optimal filter gain $L$ chosen to minimize the estimation error variance with unbiased constraint $E\left[\hat{x}_{k \mid k-1}\right]=E\left[x_{k}\right]$ is obtained as [24]:

$$
L=\left[\begin{array}{ll}
A^{N} & M_{N}
\end{array}\right]\left[\begin{array}{c}
\left(C_{N}^{T} \Pi_{N}^{-1} C_{N}\right)^{-1} C_{N}^{T} \Pi_{N}^{-1} \\
Q_{N} \Pi_{N}^{-1}\left\{I-C_{N}\left(C_{N}^{T} \Pi_{N}^{-1} C_{N}\right)^{-1} C_{N}^{T} \Pi_{N}^{-1}\right\}
\end{array}\right],
$$

where:

$$
\begin{aligned}
M_{N}= & {\left[\begin{array}{cccc}
A^{N-1} G & \cdots & A G & G
\end{array}\right], \Pi_{N}=G_{N} Q_{N} G_{N}^{T}+R_{N}, } \\
Q_{N}= & \operatorname{blkdiag}(\overbrace{Q, \cdots, Q}^{N}), R_{N}=\operatorname{blkdiag}(\overbrace{R, \cdots, R}^{N}), \\
C_{N}= & {\left[\begin{array}{c}
C \\
C A \\
C A^{2} \\
\vdots \\
C A^{N-1}
\end{array}\right], G_{N}=\left[\begin{array}{ccccc}
0 & 0 & \cdots & 0 & 0 \\
C G & 0 & \cdots & 0 & 0 \\
C A G & C G & \cdots & 0 & 0 \\
\vdots & \vdots & \ddots & \vdots & \vdots \\
C A^{N-2} G & C A^{N-3} G & \cdots & C G & 0
\end{array}\right] . }
\end{aligned}
$$

The optimal gain matrix $L$ for the state space model (7) and (8) can be obtained according to the following correspondences: $A \rightarrow \Phi, G \rightarrow I, C \rightarrow H, Q \rightarrow Q^{t i}$, and $R \rightarrow R^{s}$.

The estimates of the amplitude and phase-angle of the $m$-th harmonic components, $\hat{a}_{m, k}$ and $\hat{\theta}_{m, k}$ can be obtained from the estimated state $\hat{x}_{k \mid k-1}$ as follows:

$$
\begin{aligned}
& \hat{a}_{m, k}=\sqrt{\left(\hat{x}_{2 m-1, k \mid k-1}\right)^{2}+\left(\hat{x}_{2 m, k \mid k-1}\right)^{2}} \\
& \hat{\theta}_{m, k}=\tan ^{-1}\left(\frac{\hat{x}_{2 m, k \mid k-1}}{\hat{x}_{2 m-1, k \mid k-1}}\right)-\omega m \Delta t k .
\end{aligned}
$$

\section{Performance Evaluation}

In this section, some simulations are done to evaluate the performance of the proposed method. Consider the following voltage signal harmonics:

$$
\begin{aligned}
y(t)= & \alpha \cos (\omega t+60)+0.1 \cos (3 \omega t+15)+0.095 \cos (5 \omega t-10)+0.035 \cos (7 \omega t+40) \\
& +0.014 \cos (9 \omega t+30)+0.01 \cos (11 \omega t+50)+0.02 \cos (13 \omega t+70)+v(t),
\end{aligned}
$$

with the nominal system frequency $f=60 \mathrm{~Hz}$ and the sampling frequency of the data acquisition unit as $1.62 \mathrm{kHz}$ (27 samples per cycle). 
To illustrate the performance of the proposed method, optimal FIR filter, the 14-state time-varying (TV) Kalman filter and the adaptive Kalman filter are implemented for the state space model (7) and (8), and the simulation results are compared.

\subsection{Case 1: Time-Invariant Harmonic Components}

Firstly, to verify the estimation performance of the proposed algorithm, the proposed method is compared with the TV Kalman filter for the signal that has time-invariant harmonic components. For this simulation scenario, $\alpha$ in (15) is set as 1.0, the $Q R$ ratio is set as 0.25 and the covariance of measurement noise $R$ is set as 0.05 . The waveform of the test signal is shown in Figure 1.

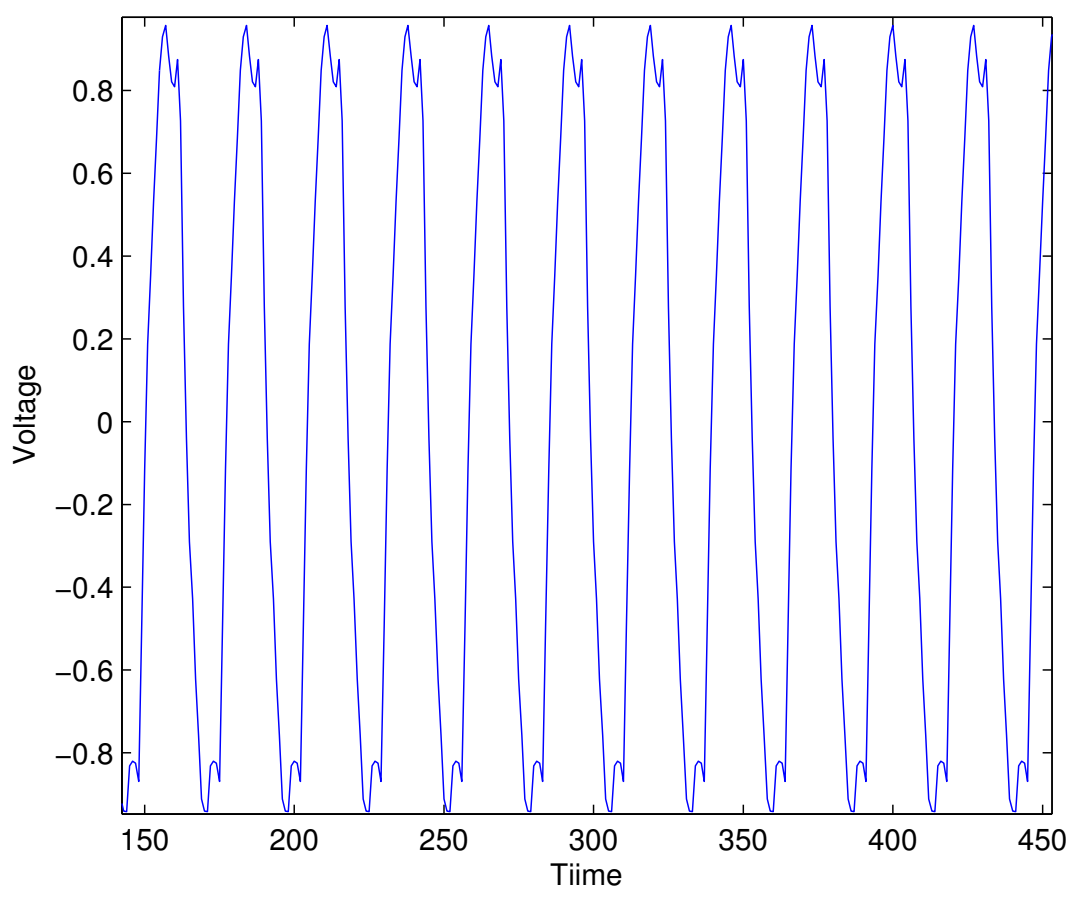

Figure 1. Waveform of test signal (Case 1).

The TV Kalman filter used in this simulation is designed as follows:

- Time update

- Project the state ahead:

$$
\hat{x}_{k \mid k-1}=A \hat{x}_{k-1 \mid k-1},
$$

- Project the error covariance ahead:

$$
P_{k \mid k-1}=A P_{k-1 \mid k-1} A^{T}+Q,
$$

- Measurement update

- Compute the filter gain:

$$
K_{k}=P_{k \mid k-1} C^{T}\left(C P_{k \mid k-1} C^{T}+R\right)^{-1},
$$

- Update estimation with measurement:

$$
\hat{x}_{k \mid k}=\hat{x}_{k \mid k-1}+K_{k}\left(y_{k}-C \hat{x}_{k \mid k-1}\right),
$$

- Update the error covariance:

$$
P_{k \mid k}=\left(I-K_{k} C\right) P_{k \mid k-1} .
$$


The simulation parameters are set as follows: the horizon size of the optimal FIR filter $N$ is selected as 14, and the initial state and initial estimation error variance for the TV Kalman filter design are set as $x_{0}=\left[\begin{array}{llll}1 & 1 & \cdots & 1\end{array}\right]^{T}$ and $P_{0}=10 \times I$, respectively.

The estimated magnitudes and its root-mean-square (rms) estimation errors of each harmonic components of two approaches are compared in Figures 2 and 3 and Table 1, respectively. As seen in these results, the proposed method and TV Kalman filter-based method provide reliable estimation results.

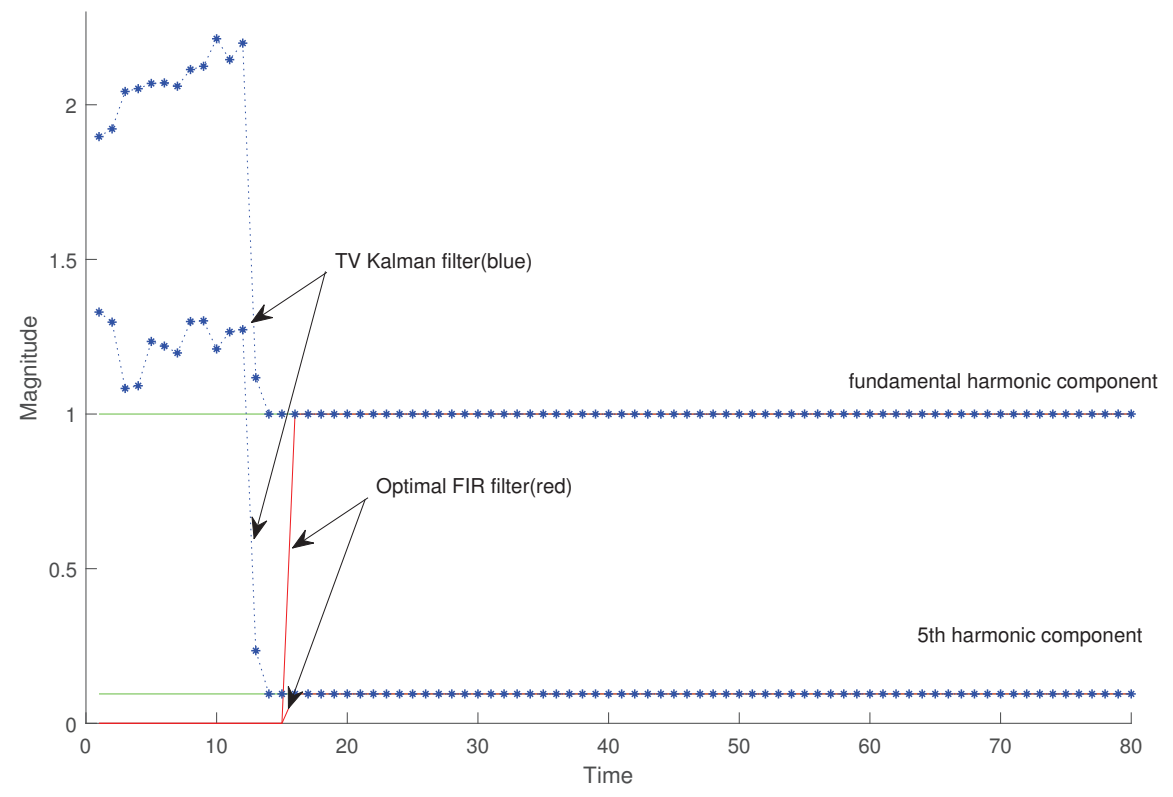

Figure 2. Estimated magnitude of fundamental and fifth harmonic components. TV, time-varying.

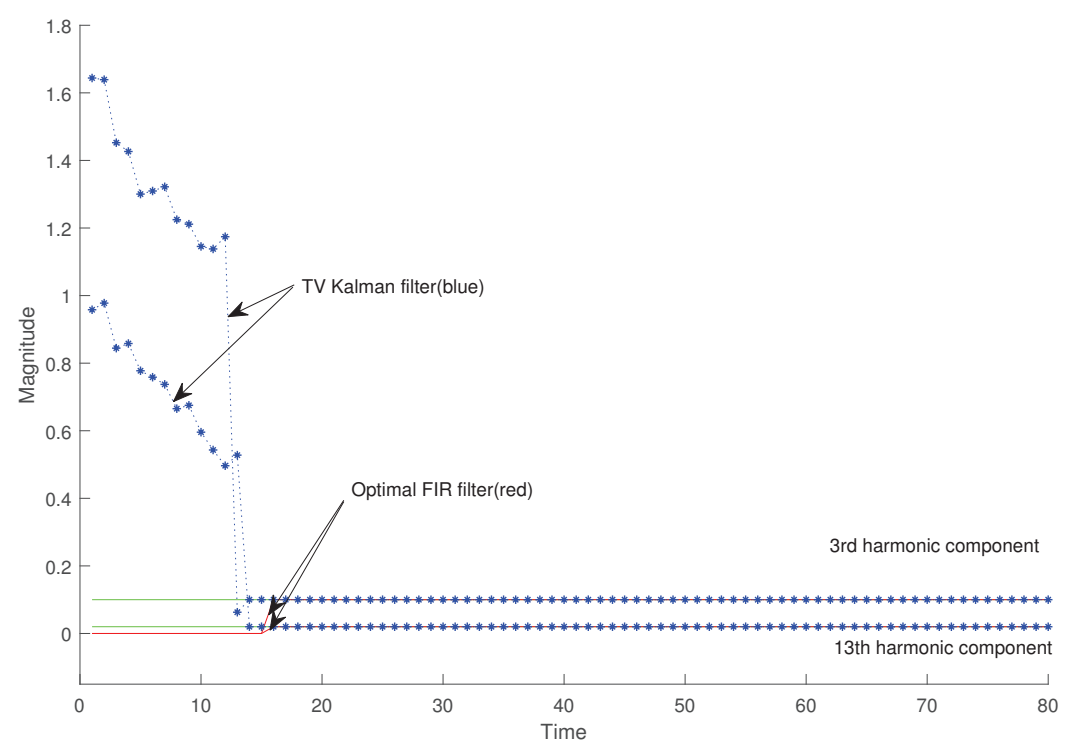

Figure 3. Estimated magnitude of third and 13th harmonic components.

Table 1. The rms estimation errors $\left(\times 10^{-3}\right)$.

\begin{tabular}{cccccccc}
\hline Filter & Fundamental & 3rd & 5th & 7th & 9th & 11th & 13th \\
\hline Optimal FIR filter & 0.1463 & 0.0578 & 0.0439 & 0.0410 & 0.0385 & 0.0382 & 0.0422 \\
TV Kalman filter & 0.1333 & 0.0544 & 0.0421 & 0.0401 & 0.0376 & 0.0372 & 0.0334 \\
\hline
\end{tabular}


However, since the optimal FIR filter used in the proposed method is a time-invariant filter, it is expected that the proposed method will have better computational efficiency than the TV Kalman filter-based method. In order to compare the computational efficiency, the average computation times per iteration of the two algorithms are compared in Table 2.

Table 2. The comparison of the average computation time.

\begin{tabular}{ccc}
\hline Filter & Optimal FIR Filter & TV Kalman Filter \\
\hline Computation time $(\mu \mathrm{s})$ & 0.971 & 1.517 \\
\hline
\end{tabular}

As we expected, the computation time of the proposed method is about $23 \%$ less than that of TV Kalman filter-based method.

\subsection{Case 2: Time-Varying Harmonic Components}

Secondly, to verify the robustness and tracking performance, we consider the signal with timevarying harmonic components. For this scenario, $\alpha$ in (15) is set as:

$$
\alpha=\left\{\begin{array}{cc}
1.0, & 0 \leq k \leq 299 \\
0.2, & 300 \leq k \leq 599 \\
1.2, & 600 \leq k \leq 900
\end{array}\right.
$$

The test signal with time-varying harmonic components is shown in Figure 4.

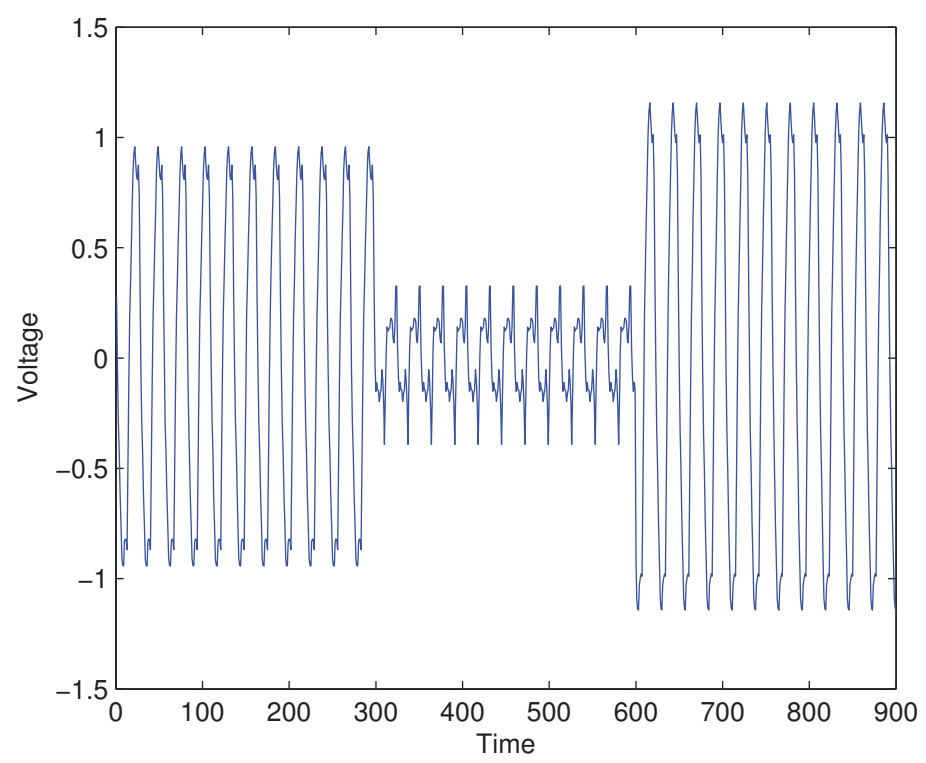

Figure 4. Waveform of test signal (Case 2).

In order to verify the efficiency of the use of FIR-structured filter, the proposed method and TV Kalman filter-based method are compared for the test signal with time-varying harmonic components.

Figures 5-7 and Table 3 show the estimated magnitude and its rms estimation errors of the fundamental component with various $Q R$ ratios $(R=0.05$, constant). As seen in these results, there are no significant changes in the estimate of the proposed method, whereas that of the TV Kalman filter cannot converge to the real magnitude. These results verify that the FIR-structured filter-based method is less sensitive to the covariance matrix $Q$. Thus, it can be said that the proposed method is more robust than the Kalman filter-based approach. The oscillatory behavior of two methods in Figures $5-7$ is due to the measurement noise $v_{k}$ and inaccurate process noise covariance matrix $Q$. 


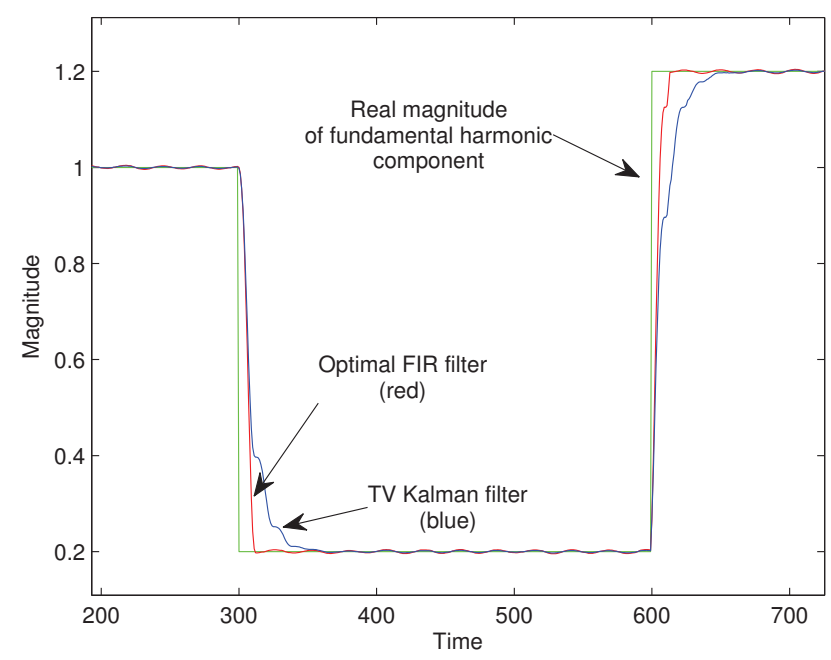

Figure 5. Estimated magnitude of the fundamental component with $Q / R=0.025$.

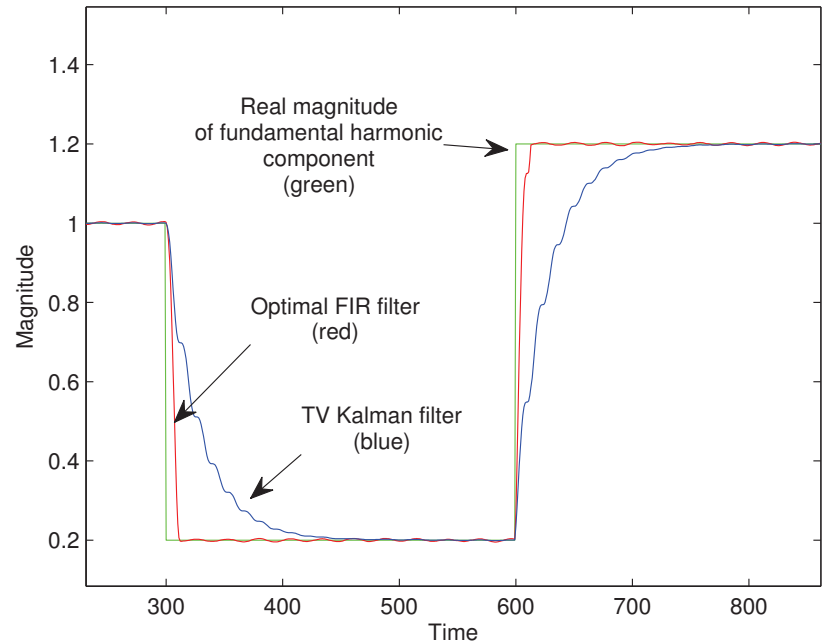

Figure 6. Estimated magnitude of the fundamental component with $Q / R=0.0025$.

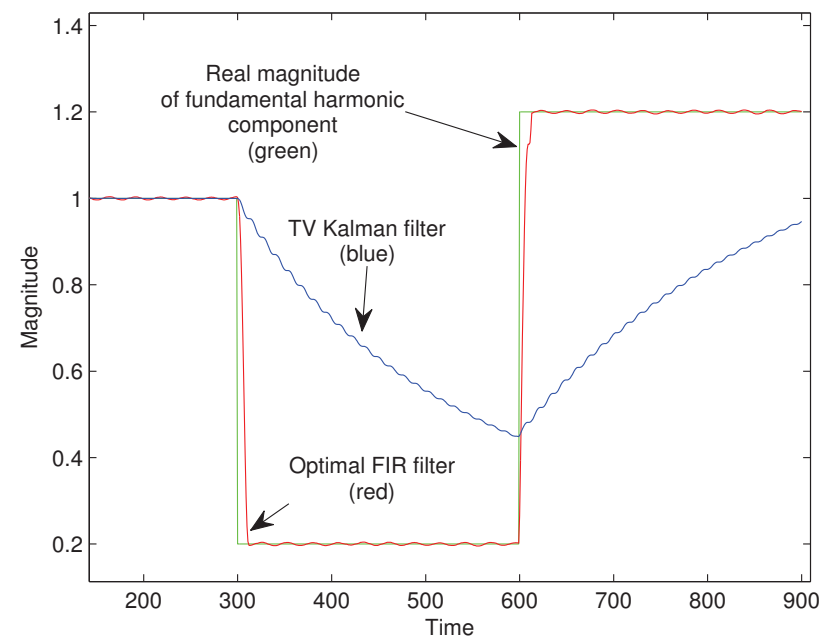

Figure 7. Estimated magnitude of the fundamental component with $Q / R=0.00025$. 
Table 3. The rms estimation errors with various $Q / R$.

\begin{tabular}{cccc}
\hline Filter & $Q / R=\mathbf{0 . 0 2 5} \mathbf{h}$ & $Q / R=\mathbf{0 . 0 0 2 5} \mathbf{h}$ & $Q / R=\mathbf{0 . 0 0 0 2 5} \mathbf{h}$ \\
\hline Optimal FIR filter & 0.0936 & 0.0966 & 0.0973 \\
TV Kalman filter & 0.1041 & 0.1609 & 0.2788 \\
\hline
\end{tabular}

In addition, the proposed method is compared with the adaptive Kalman filter-based method to verify the robustness and convergence ability. The adaptive Kalman filter has the same structure as the TV Kalman filter, and the process and measurement covariance matrices $Q$ and $R$ are tuned each iteration as follows [23]:

$$
\begin{aligned}
R_{k} & =r_{k} I \\
r_{k} & =\beta r_{k-1}+(1-\beta) \hat{r}_{k \prime} \\
\hat{r}_{k} & =f\left(e_{k}^{2}-C P_{k+1 \mid k} C^{T}\right) . \\
Q & =q_{k} I, \\
q_{k} & =\beta q_{k-1}+(1-\beta) \hat{q}_{k \prime} \\
\hat{q}_{k} & =f\left(\frac{e_{k}^{2}-\left(C P_{k+1 \mid k} C^{T}+R_{k}\right)}{C C^{T}}\right),
\end{aligned}
$$

where $\beta$ is a positive scalar and predictor error $e_{k}$ and function $f(\cdot)$ are defined as follows:

$$
\begin{aligned}
e_{k} & =y_{k}-C \hat{x}_{k \mid k-1} \\
f(n) & = \begin{cases}n, & \text { if } n \geq 0 \\
0, & \text { otherwise }\end{cases}
\end{aligned}
$$

To design the adaptive Kalman filter, parameters are set as $q_{0}=0.25, r_{0}=1, e_{0}=0$ and $\beta=0.5$, respectively. The initial state and initial estimation error variance are set the same as the TV Kalman filter parameters.

The rms estimation errors of the proposed method and the adaptive Kalman filter-based method are shown in Table 4. From this result, the estimation error of the proposed method is lesser than that of the adaptive Kalman filter-based approach.

Table 4. The rms estimation errors.

\begin{tabular}{cccccccc}
\hline Filter & Fundamental & 3rd & 5th & 7th & 9th & 11th & 13th \\
\hline Optimal FIR filter & 0.0935 & 0.0238 & 0.0113 & 0.0038 & 0.0050 & 0.0062 & 0.0054 \\
TV Kalman filter & 0.1027 & 0.0260 & 0.0146 & 0.0073 & 0.0090 & 0.0087 & 0.0075 \\
\hline
\end{tabular}

Figures 8 and 9 show the estimated magnitude of harmonic components of the proposed method and the adaptive Kalman filter-based method. As seen in these figures, the estimate of the proposed method converges to the real magnitude of each harmonic component faster than that of the adaptive Kalman filter-based approach. Additionally, it is expected that the computation time of the proposed method will be less than that of adaptive Kalman filter-based approach, because the adaptive Kalman filter needs to calculate $Q$ and $R$ per iteration. In Table 5, the average computation times per iteration of the two algorithms are compared. 

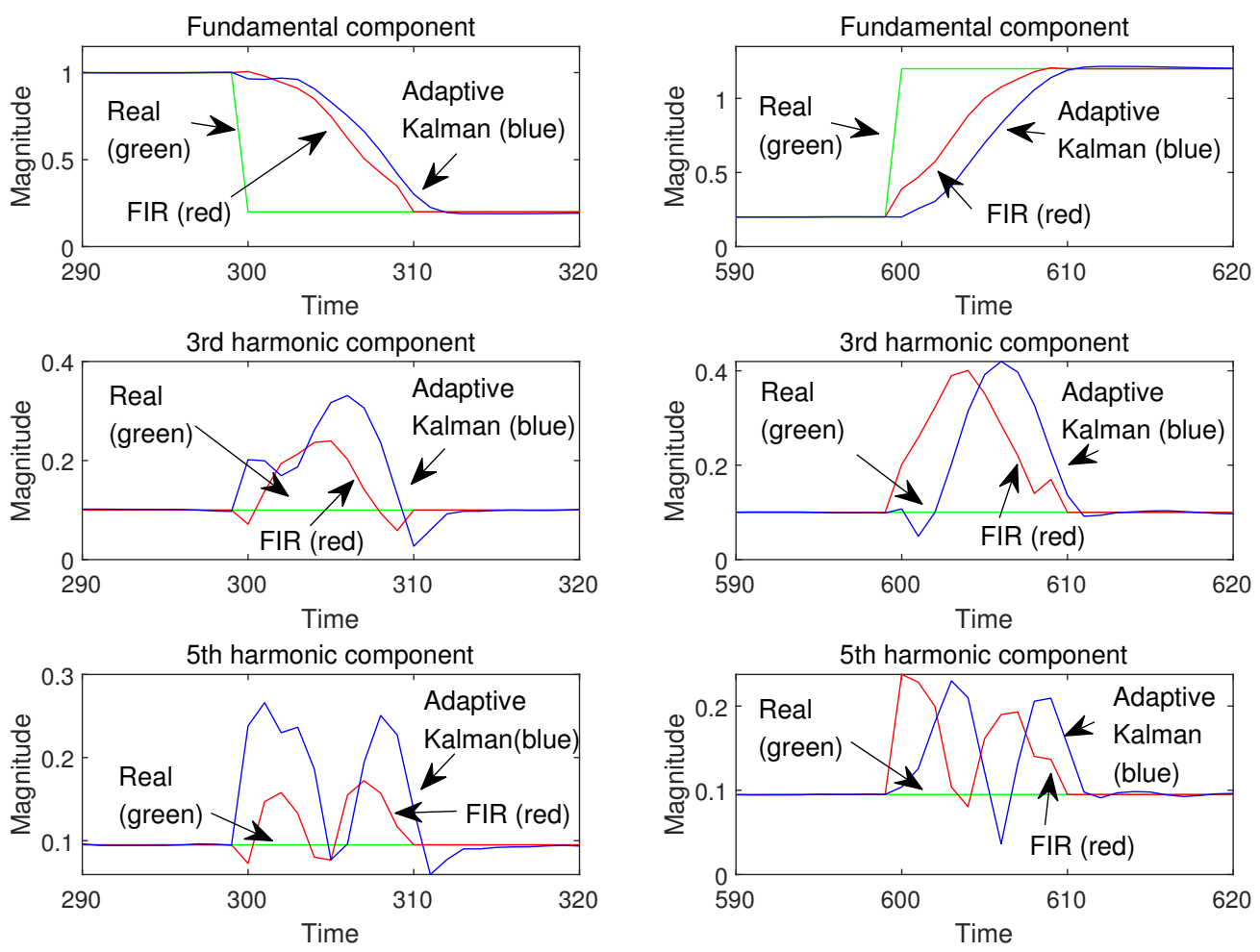

Figure 8. Real and estimated magnitude of the fundamental, third and fifth harmonic components.
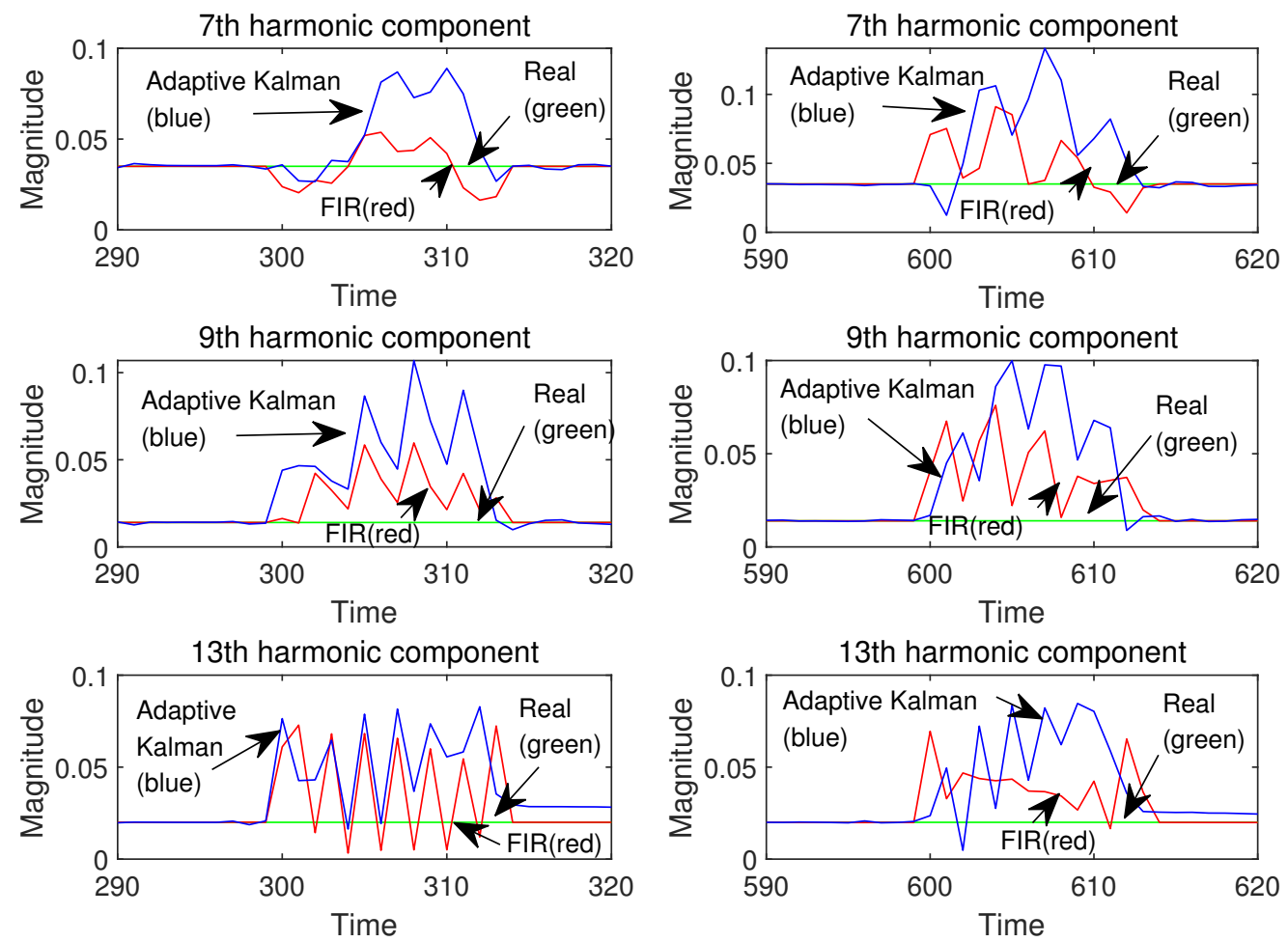

Figure 9. Real and estimated magnitude of 7 th, 9 th and 13th harmonic components.

As seen in the result, the computation time of the proposed method is $72.84 \%$ less than that of adaptive Kalman filter-based method. 
Table 5. The comparison of the average computation time.

\begin{tabular}{ccc}
\hline Filter & Optimal FIR Filter & Adaptive Kalman Filter \\
\hline Computation time $(\mu \mathrm{s})$ & 0.972 & 3.579 \\
\hline
\end{tabular}

\section{Estimation of the Power Conditioning System Harmonic Components}

Due to the high efficiency and environmental acceptability, the solid oxide fuel cell (SOFC) is considered as an attractive power source in the distributed generation power system [29].

As a flexible and controllable distributed generation source, SOFC is required to fill the power gap to reduce the intervention from and impact to the utility grid. The SOFC is connected to the grid through power electronic converters to improve the system reliability and efficiency. To provide power to the power grid, a power conditioning system (PCS) is required to interface between the SOFC and power grid to convert the DC voltage of SOFC into AC voltage, as shown in Figure 10. From the configuration of the grid-connected SOFC in Figure 10, there are two subsystems, which are the SOFC and PCS, respectively. The PCS consists of a DC/DC boost converter and a DC/AC inverter. The DC/DC converter is to boost the output voltage of SOFC to a higher voltage in the DC link, and the $\mathrm{DC} / \mathrm{AC}$ inverter is to convert DC voltage into AC voltage to supply the power to the load and the power grid. From the point of SOFC, it should not only follow the power demand as quickly as possible, but also keep working safely. However, the power electronic converters are sensitive to grid disturbances. Therefore, it is necessary to design proper control strategies to keep the system stable under any disturbance and parameter variations in the distribution system. The current control strategy is a commonly-used control method in a grid-connected system to control the amount of output power in the DC/AC inverter.

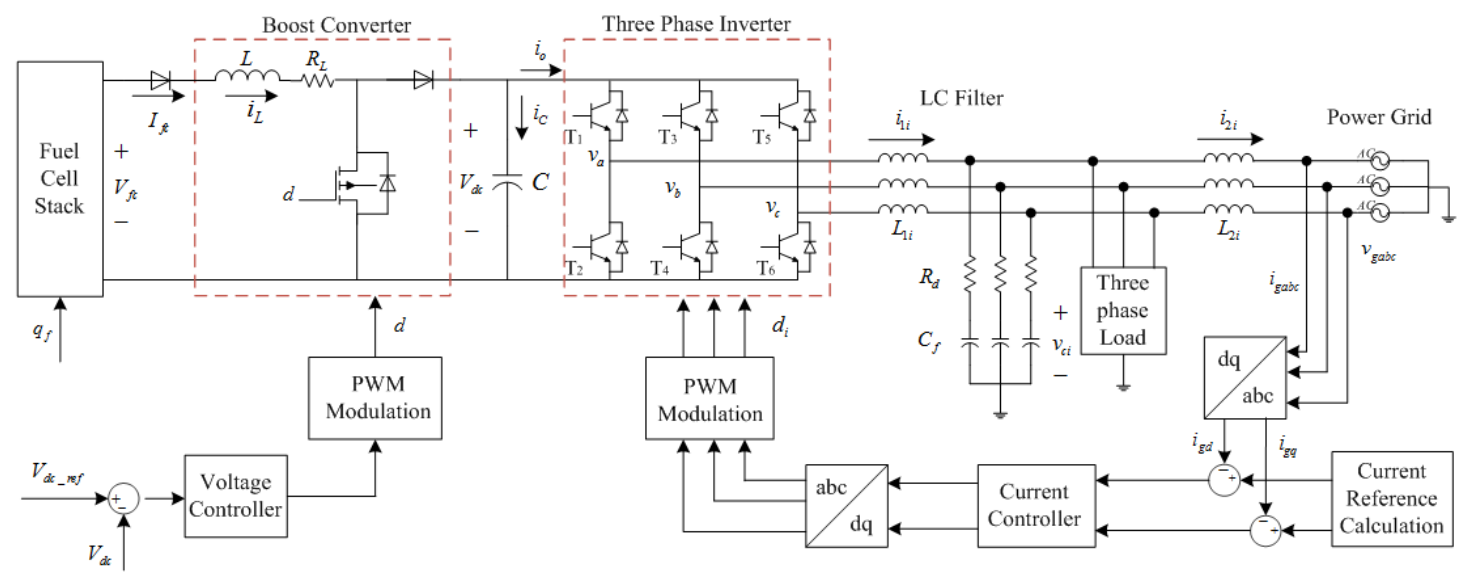

Figure 10. System configuration of the power conditioning system.

To make sure that a high quality of the current is provided to the load and the power grid side, it is important to measure the total effect of harmonic components. The total harmonic distortions (THDs) can give the information of the harmonic components in the current signal. THDs of the output current of the inverter may exceed the required standard, which is less than $5 \%$. Thus, it is important to have more precise components of harmonics to estimate the THDs, and the proposed algorithm can give a good solution to estimate the harmonic components of the PCS current signal and THDs.

The current signal of PCS (Phase A) is shown in Figure 11. The sampling frequency of the data acquisition is $1.67 \mathrm{kHz}$, and the white Gaussian random process noises are added to the all measurements with measurement noise covariance $R=15$. To design the filters, the measurement noise covariance for the filter is set as 10, the horizon size of the FIR filter $N$ is set as 21 and the initial state and initial estimation error variance are set as $x_{0}=\left[\begin{array}{llll}1 & 1 & \cdots & 1\end{array}\right]^{T}$ and $P_{0}=1000 \times I$, respectively. 


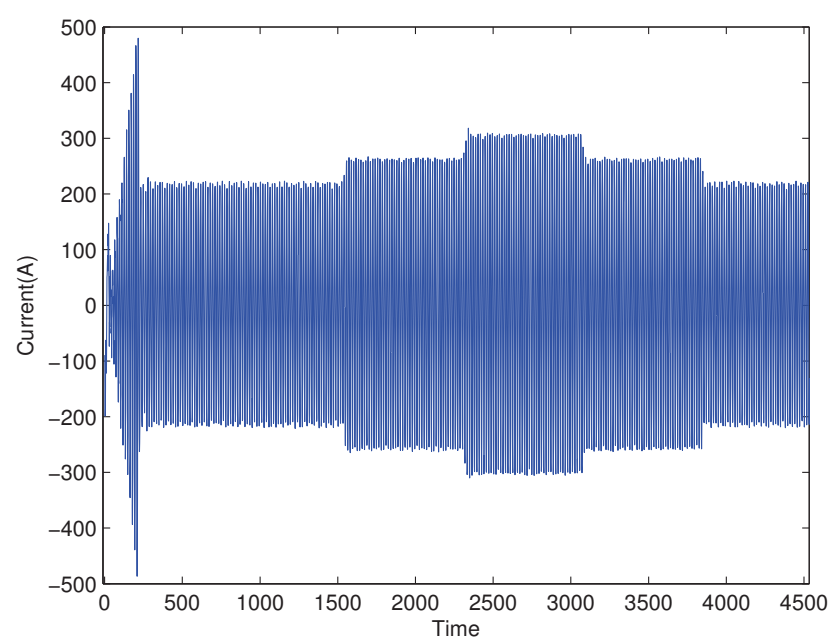

Figure 11. Current signal of power conditioning system (PCS) (Phase A).

The estimated fundamental magnitudes of the proposed method and TV Kalman filter-based method with various $Q R$ ratios are shown in Figures 12 and 13. As seen in these figures, the proposed method gives a more robust estimate than the TV Kalman filter-based method for PCS harmonic components' estimation. These results verify the robustness of the proposed method in power system harmonic estimation.

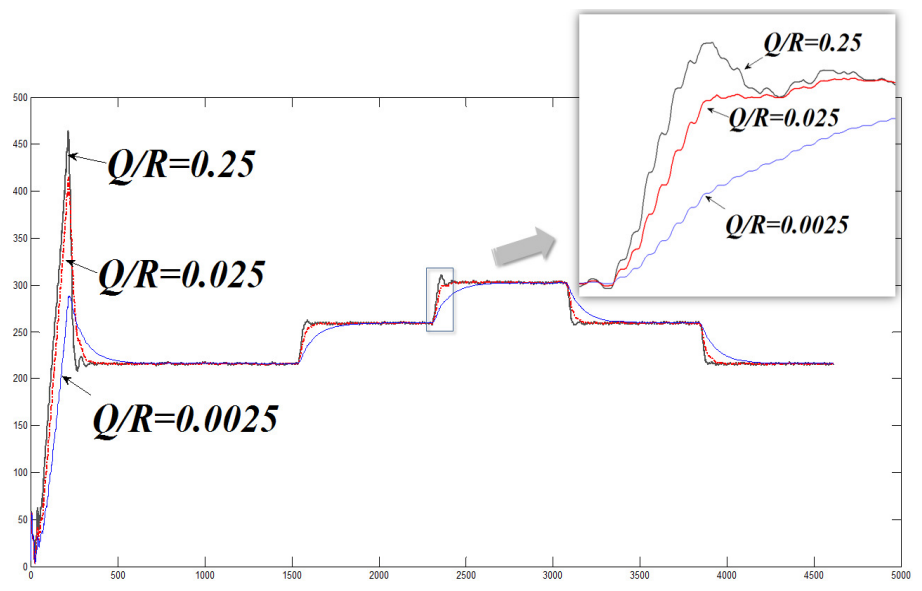

Figure 12. Estimated magnitude of the fundamental component: TV Kalman filter with various $Q / R$.

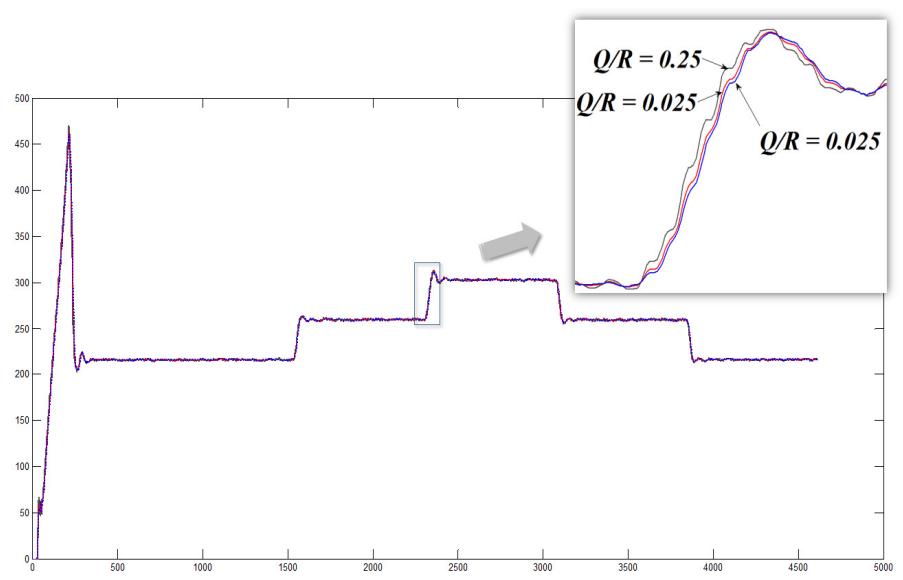

Figure 13. Estimated magnitude of the fundamental component: optimal FIR filter with various $Q / R$. 


\section{Conclusions}

In this paper, a robust estimation technique was introduced to estimate the magnitude and phase of the harmonic components of the power system. In order to design the robust estimator, the optimal FIR filter was applied to the state space representation of the noisy current or voltage signals. Since the optimal FIR filter used in the proposed method consisted of a linear combination of a finite number of past measurements, the proposed method could avoid the divergence problem that might arise in the case of Kalman filter-based approaches. The proposed method could provide more robust estimation of uncertainties and numerical errors and a faster tracking ability compared with the Kalman filter-based approaches, because the FIR-structured filter was used to estimate the harmonic components. Since the optimal FIR filter used in this approach is not use any a priori information of the initial state, the proposed harmonic estimation method could prevent errors from the unknown initial state. Moreover, since the optimal FIR filter used in this approach is a time-invariant filter, the proposed method could give better computation efficiency than the Kalman filter-based approaches, which use the time-varying Kalman filter. Through the simulation results, it was shown that the proposed method is much less sensitive for the process noise than the Kalman filter-based approaches. Furthermore, it was verified that the proposed method is more robust and has better tracking performance and computational efficiency than the Kalman filter-based approaches by comparison with the time-varying Kalman filter and the adaptive Kalman filter-based approaches. Moreover, it was shown that the proposed method could be applied to the real power system by employing the proposed method to estimate the harmonic components of PCS.

Author Contributions: Methodology, B.K.; Software, B.K.; Validation, B.K. and S.H.; Formal Analysis, B.K.; Resources, K.Y.L.; Writing—Original Draft Preparation, B.K.; Writing—Review \& Editing, S.H.; Supervision, K.Y.L.

Funding: This research received no external funding.

Acknowledgments: This study is supported by Kangwon National University. This work is also supported by "Human Resources Program in Energy Technology" of the Korea Institute of Energy Technology Evaluation and Planning (KETEP), granted financial resource from the Ministry of Trade, Industry and Energy, Republic of Korea. (No. 20174030201660).

Conflicts of Interest: The authors declare no conflict of interest.

\section{References}

1. Chen, R.; Lin, T.; Bi, R.; Xu, X. Novel Strategy for Accurate Locating of Voltage Sag Sources in Smart Distribution Networks with Inverter-Interfaced Distributed Generators. Energies 2017, 10, 1855. [CrossRef]

2. Rafael, C.; Aurelio, M.; Olimpo, A. Time-Domain Voltage Sag State Estimation Based on the Unscented Kalman Filter for Power Systems with Nonlinear Components. Energies 2018, 11, 1411. [CrossRef]

3. Yilmatz, A.S.; Alkan, A.; Asyali, M.H. Application of parametric spectral estimation methods on detection of power system harmonics. Electr. Power Syst. Res. 2008, 78, 683-693. [CrossRef]

4. Ray, P.; Subudhi, B. Ensemble-Kalman-filter-based power system harmonic estimation: 3216-3224. IEEE Trans. Instrum. Meas. 2012, 61, 1153-1160. [CrossRef]

5. Chen, C.I.; Chen, Y.C. Comparative study of harmonic and inter harmonic estimation methods for stationary and time-varying signals. IEEE Trans. Ind. Electron. 2014, 61, 397-404. [CrossRef]

6. Rico, J.J.; Madrigal, M.; Acha, E. Dynamic harmonic evolution using the extended harmonic domain. IEEE Trans. Power Deliv. 2003, 18, 587-594. [CrossRef]

7. Vargas, U.; Ramirez, A.; Lazaroiu, G.C. Lazaroiu, Flexible extended harmonic domain approach for transient state analysis of switched systems. Electr. Power Syst. Res. 2018, 115, 40-47. [CrossRef]

8. Singh, S.K.; Sinha, N.; Goswami, A.K.; Sinha, N. Several variants of Kalman Filter algorithm for power system harmonic estimation. Electr. Power Energy Syst. 2016, 78, 793-800. [CrossRef]

9. IEC Std. 61000-4-7. General Guide on Harmonics and Inter Harmonics Measurements and Instrumentation for Power Supply Systems and Equipment Connected Thereto; International Electrotechnical Commission: Geneva, Switzerland, 2009. 
10. Singh, G.K. Power system harmonics research: A survey. Eur. Trans. Electr. Power 2009, 19, 151-172. [CrossRef]

11. Jain, S.K.; Singh, S.N. Fast Harmonic Estimation of Stationary and Time-Varying Signals Using EA-AWNN. IEEE Trans. Instrum. Meas. 2013, 62, 335-343. [CrossRef]

12. Agrez, D. Weighted multipoint interpolated DFT to improve amplitude estimation of multifrequency signal. IEEE Trans. Instrum. Meas. 2002, 51, 287-292. [CrossRef]

13. Saiz, V.M.M.; Guadalupe, J.B. Application of Kalman filtering for continuous realtime tracking of power system harmonics. Proc. Gener. Transm. Distrib. 1998, 144, 13-20. [CrossRef]

14. Marei, M.I.; Shatshat, R.E. Fast Envelope Estimation Technique for Monitoring Voltage Fluctuations. J. Electr. Eng. Technol. 2007, 2, 445-451. [CrossRef]

15. Jo, S.H.; Son, S.E.; Lee, S.; Park, J.W. Kalman-filter-based multilevel analysis to estimate electric load composition. IEEE Trans. Ind. Electron. 2012, 59, 4263-4271. [CrossRef]

16. Kennedy, K.; Lightbody, G.; Yacamini, R. Power system harmonic analysis using the Kalman filter. In Proceedings of the 2003 IEEE Power Engineering Society General Meeting, Toronto, ON, Canada, 13-17 July 2003.

17. Liu, S. An adaptive Kalman filter for dynamic estimation of harmonic signals. In Proceedings of the 8th International Conference on Harmonics and Quality of Power, Athens, Greece, 14-16 October 1998.

18. Yu, K.K.C.; Watson, N.R.; Arrillaga, J. An adaptive Kalman filter for dynamic harmonic state estimation and harmonic injection tracking. IEEE Trans. Power Deliv. 2005, 2, 1577-1584. [CrossRef]

19. Rosendo, J.A.; Gomez, A. Self tunning of Kalman filters for harmonic computation. IEEE Trans. Power Deliv. 2006, 21, 501-503.

20. Chen, C.I.; Chang, G.W.; Hong, R.C.; Li, H.M. Extended real model of Kalman filter for time-varying harmonics estimation. IEEE Trans. Power Deliv. 2010, 25, 17-26. [CrossRef]

21. Nayak, P.; Jena, S. Estimation of Harmonics in Microgrid using Unscented Kalman Filter. Int. J. Pure Appl. Math. 2017, 114, 73-81.

22. Zanni, L.; Le Boudec, J.Y.; Cherkaoui, R.; Paolone, M. A Prediction-Error Covariance Estimator for Adaptive Kalman Filtering in Step-Varying Processes: Application to Power-System State Estimation. IEEE Trans. Control Syst. Technol. 2017, 25, 1683-1697. [CrossRef]

23. Reddy, R.G.; Nakka, S.; Sonam, S.K. Estimation Of Power Harmonics Using Kalman Filter. Int. J. Res. Appl. Sci. Eng. Technol. 2017, 5, 1251-1257.

24. Kwon, W.H.; Han, S. Receding Horizon Control: Model Predictive Control for State Models; Springer: Heidelberg/Berlin, Germany, 2005.

25. Kwon, B.K.; Han, S.; Kwon, O.K.; Kwon, W.H. Minimum variance FIR smoothers for discretetime state space models. IEEE Signal Proc. Lett. 2007, 14, 557-560. [CrossRef]

26. Kwon, B.K.; Han, S. Least-Mean-Square Receding Horizon Estimation. Math. Probl. Eng. 2012, $2012,1-19$. [CrossRef]

27. Kwon, B.K.; Quan, Z.; Han, S. A Robust Fixed-lag Receding Horizon Smoother for Uncertain State Space Models. Int. J. Adapt. Control Signal Proc. 2015, 29, 1354-1366. [CrossRef]

28. Kwon, B.K.; Quan, Z.; Han, S. Improved Receding Horizon Fourier Analysis for Quasi-periodic Signals. J. Electr. Eng. Technol. 2016, 12, 378-384. [CrossRef]

29. Wu, G.; Lee, K.Y. Sliding Mode Control of a Hybrid Fuel cell-Battery Power System. In Proceedings of the 9th IFAC symposium on Control of Power and Energy Systems, New Delhi, India, 9-11 December 2015.

(c) 2018 by the authors. Licensee MDPI, Basel, Switzerland. This article is an open access article distributed under the terms and conditions of the Creative Commons Attribution (CC BY) license (http://creativecommons.org/licenses/by/4.0/). 